\title{
Search Directions in Infeasible Newton's Method for Computing Weighted Analytic Center for Linear Matrix Inequalities
}

\author{
Shafiu Jibrin*, Ibrahim Abdullahi \\ Department of Mathematics, Faculty of Science, Federal University, Dutse, Nigeria \\ Email address: \\ shafiuj@fud.edu.ng (S. Jibrin), ibrahim.abdullahi@fud.edu.ng (I. Abdullahi) \\ ${ }^{*}$ Corresponding author
}

To cite this article:

Shafiu Jibrin, Ibrahim Abdullahi. Search Directions in Infeasible Newton's Method for Computing Weighted Analytic Center for Linear Matrix Inequalities. Applied and Computational Mathematics. Vol. 8, No. 1, 2019, pp. 21-28. doi: 10.11648/j.acm.20190801.14

Received: February 11, 2019; Accepted: March 22, 2019; Published: April 22, 2019

\begin{abstract}
Four different search directions for Infeasible Newton's method for computing the weighted analytic center defined by a system of linear matrix inequality constraints are studied. Newton's method is applied to find the weighted analytic center and the starting point can be infeasible, that is, outside the feasible region determined by the linear matrix inequality constraints. More precisely, Newton's method is used to solve system of equations given by the KKT optimality conditions for the weighted analytic center. The search directions for the Newton's method considered are the ZY, ZY+YZ, $Z^{-1}$ and NT methods that have been used in semidefinite programming. Backtracking line search is used for the Newton's method. Numerical experiments are used to compare these search direction methods on randomly generated test problems by looking at the iterations and time taken to compute the weighted analytic center. The starting points are picked randomly outside the feasible region. Our numerical results indicate that $\mathrm{ZY}+\mathrm{YZ}$ and $\mathrm{ZY}$ are the best methods. The $\mathrm{ZY}+\mathrm{YZ}$ method took the least number of iterations on average while $\mathrm{ZY}$ took the least time on average and they handle weights better than the other methods when some of the weights are very large relative to the other weights. These are followed by NT method and then $Z^{-1}$ method.
\end{abstract}

Keywords: Linear Matrix Inequalities, Weighted Analytic Center, Newton’s Method, Semidefinite Programming

\section{Introduction}

Consider a system of linear matrix inequality (LMI) constraints given below:

$$
A^{(j)}(x):=A_{0}^{(j)}+\sum_{i=1}^{n} x_{i} A_{i}^{(j)} \succeq 0, \quad(j=1,2, \ldots, q),
$$

where $x \in \mathrm{IR}^{n}$ is a variable and each $A_{i}^{(j)}$ is a $m_{j} \times m_{j}$ symmetric matrix for $i=0,1, \ldots, n$. LMI constraints have applications in a variety of areas including engineering, geometry and statistics [1, 9]. Assume that feasible determined by the constraints is bounded and has a nonempty interior.

Let $\mathcal{R}$ denote the feasible region defined by the inequalities (1). Given $\omega>0$, define the barrier function $\varphi_{\omega}(x): \mathrm{IR}^{n} \rightarrow \mathrm{IR}$ by:

$$
\phi_{\omega}(x)= \begin{cases}\sum_{j=1}^{q} \omega_{j} \log \operatorname{det}\left[\left(A^{(j)}(x)\right)^{-1}\right] & \text { if } x \in \operatorname{int}(\mathcal{R}) \\ \infty & \text { otherwise }\end{cases}
$$

The weighted analytic center for the system (1) was introduced by Pressman and Jibrin [7], and discussed in a paper by Jibrin and Swift [5]. It is defined by:

$$
x_{a c}(\omega)=\operatorname{argmin}\left\{\varphi_{\omega}(x) \mid x \in \mathrm{IR}^{n}\right\}
$$

This is a more general form of the determinant maximization problem considered by Vandenberghe et al. [11]. In the special case of linear constraints, weighted analytic center has been studied extensively in the past [3]. This definition of weighted analytic center for LMIs extends that of linear constraints studied by Atkinson and Vaidya [3].

Jibrin presents Infeasible Newton's method for finding 
weighted analytic center for the system (1) [4]. A feasible starting point is not required to start the method. In this paper, four search directions for the method, namely: ZY, $\mathrm{ZY}+\mathrm{YZ}, Z^{-1}$ and NT methods are compared in computing the weighted analytic center. These search directions have been used in the past in the problem of semidefinite programming [2]. The $\mathrm{ZY}+\mathrm{YZ}$ method took the least number of iterations on average while $Z Y$ took the least time on average and they handle weights better than the other methods when some of the weights are very large relative to the other weights. These are followed by NT method and then $Z^{-1}$ method. The results agree with what is known in semidefinite programming, where $\mathrm{ZY}+\mathrm{YZ}$ and $\mathrm{ZY}$ are found to be more efficient among the four methods.

\section{Infeasible Newton's Method for Computing Weighted Analytic Center}

This section briefly describes infeasible Newton's method for computing weighted analytic center for the system (1) given by Jibrin in an earlier paper [4].

The optimality conditions for computing the weighted analytic center $x_{a c}(\omega)$ are given by:

$$
\begin{gathered}
A_{0}^{(j)}+\sum_{i=1}^{n} x_{i} A_{i}^{(j)}-Y^{(j)}=0,(j=1, \ldots, q) \\
\sum_{j=1}^{q} A_{i}^{(j)} \cdot Z^{(j)}=0, \quad(i=1, \ldots, n) \\
Z^{(j)} Y^{(j)}=\omega_{j} I_{m_{j}}, \quad(j=1, \ldots, q) \\
Y^{(j)} \succ 0,(j=1, \ldots, q) \\
Z^{(j)} \succ 0, \quad(j=1, \ldots, q)
\end{gathered}
$$

Let

$$
\begin{gathered}
z^{(j)}=\operatorname{vec} Z^{(j)} \\
y^{(j)}=\operatorname{vec} Y^{(j)} \\
B^{(j)}=\left[\begin{array}{c}
\left(\operatorname{vec} A_{1}^{(j)}\right)^{T} \\
\vdots \\
\left(\operatorname{vec} A_{n}^{(j)}\right)^{T}
\end{array}\right] \\
R_{p}^{(j)}=Y^{(j)}-A_{0}^{(j)}-m a t\left(B^{(j)}\right)^{T} x \\
R_{d}=\left[\begin{array}{c}
q \\
-\sum_{j=1}^{q} A_{1}^{(j)} \cdot Z^{(j)} \\
\vdots \\
-\sum_{j=1}^{q} A_{n}^{(j)} \cdot Z^{(j)}
\end{array}\right]
\end{gathered}
$$

$$
\begin{gathered}
R_{c}^{(j)}=\omega_{j} I_{m_{j}}-Z^{(j)} Y^{(j)} \\
r_{p}^{(j)}=\operatorname{vec} R_{p}^{(j)}=y^{(j)}-\operatorname{vec} A_{0}^{(j)}-\left(B_{j}^{(j)}\right)^{T} x \\
r_{d}=\operatorname{vec} R_{d}=-\sum_{j=1}^{q} B^{(j)} z^{(j)} \\
r_{c}^{(j)}=\operatorname{vec} R_{c}^{(j)}=\operatorname{vec}\left(\omega_{j} I_{m_{j}}\right)-\left(I_{m_{j}} \otimes Z^{(j)}\right) y^{(j)} \\
r_{c}^{(j)}=\operatorname{vec} R_{c}^{(j)}=\operatorname{vec}\left(\omega_{j} I_{m_{j}}\right)-\left(Y^{(j)} \otimes I_{m_{j}}\right) z^{(j)} \\
G\left(x, y^{(1)}, \ldots, y^{(q)}, z^{(1)}, \ldots, z^{(q)}\right)=\left[\begin{array}{c}
(1) \\
-r_{p}^{(1)} \\
\vdots \\
-r_{p}^{(q)} \\
-r_{d} \\
-r_{c}^{(1)} \\
\vdots \\
-r_{c}^{(q)}
\end{array}\right]
\end{gathered}
$$

where vec is the map that stacks the columns of a matrix on top of each other into a single vector and mat is the inverse map. Also, let

$$
\begin{gathered}
r_{p}=\left[\begin{array}{c}
r_{p}^{(1)} \\
\vdots \\
r_{p}^{(q)}
\end{array}\right], r_{c}=\left[\begin{array}{c}
r_{c}^{(1)} \\
\vdots \\
r_{c}^{(q)}
\end{array}\right] \\
y=\left[\begin{array}{c}
y^{(1)} \\
\vdots \\
y^{(q)}
\end{array}\right], z=\left[\begin{array}{c}
z^{(1)} \\
\vdots \\
z^{(q)}
\end{array}\right] \\
A=\left[B^{(1)}, \ldots, B^{(q)}\right]
\end{gathered}
$$

$$
\begin{gathered}
E=\operatorname{diag}\left(Y^{(1)} \otimes I_{m_{1}}, \ldots, Y^{(q)} \otimes I_{m_{q}}\right) \\
F=\operatorname{diag}\left(I_{m_{1}} \otimes Z^{(1)}, \ldots, I_{m_{q}} \otimes Z^{(q)}\right) \\
I=\operatorname{diag}\left(I_{m_{1}^{2}}, \ldots, I_{m_{q}^{2}}\right)
\end{gathered}
$$

Then

$$
G(x, y, z)=\left[\begin{array}{l}
-r_{p} \\
-r_{d} \\
-r_{c}
\end{array}\right]=-r
$$

where $r=\left[\begin{array}{c}r_{p} \\ r_{d} \\ r_{c}\end{array}\right]$ is the residual vector. 
The weighted analytic center $x_{a c}(\omega)$ for the system (1) is given as $x$ from the root $(x, y, z)$ of the equation

$$
G(x, y, z)=0
$$

The Newton's directions $(\Delta x, \Delta y, \Delta z)$ for the equation are found by solving the system:

$$
\begin{gathered}
M \Delta x=A E^{-1}\left(F r_{p}+r_{c}\right)-r_{d} \\
\Delta z=E^{-1}\left(F\left(r_{p}-A^{T} \Delta x\right)+r_{c}\right) \\
\Delta y=F^{-1}\left(r_{c}-E \Delta z\right)
\end{gathered}
$$

where

$$
\mathrm{M}=\mathrm{AE}^{-1} \mathrm{FA}^{\mathrm{T}}
$$

An Iteration of Infeasible Newton's Method for Computing Weighted Analytic Center.

The following is an algorithm for an iteration of Infeasible Newton's Method for Weighted Analytic Center for the LMI system (1).

Step 1: Compute Newton's direction $(\Delta x, \Delta y, \Delta z)$ using equations (7)-(9). This gives $(\Delta x, \Delta y(1), \ldots, \Delta y(q), \Delta z(1), \ldots$, $\Delta \mathrm{z}(\mathrm{q}))$.

Step 2: For each $\mathrm{j}$, determine:

$$
\begin{aligned}
& \Delta Y^{(j)}=\text { mat } \Delta y^{(j)} \\
& \Delta Z^{(j)}=\text { mat } \Delta z^{(j)}
\end{aligned}
$$

Step 3: Symmetrize $\Delta Z(j)$.

$$
\text { Replace } \Delta Z^{(j)} \text { by } \frac{1}{2}\left(\Delta Z^{(j)}+\left(\Delta Z^{(j)}\right)^{T}\right),(j=1, \ldots, q)
$$

Step 4: Do line search to get stepsize $h$.

Step 5: Update the iterates.

$$
\begin{gathered}
x \leftarrow x+h \Delta x \\
Y^{(j)} \leftarrow Y^{(j)}+h \Delta Y^{(j)},(j=1, \ldots, q) \\
Z^{(j)} \leftarrow Z^{(j)}+h \Delta Z^{(j)},(j=1, \ldots, q)
\end{gathered}
$$

Step 6: Find $(x, y, z)$ and calculate the residual vector $r(x, y, z)$.

Any point $x \in \mathrm{IR}^{n}$ can be picked as a starting point. Then, for $j=1, \ldots, q$, choose.

$$
\begin{gathered}
Y^{(j)}=\left\{\begin{array}{cc}
A^{(j)}(x) & A^{(j)}(x) \succ 0 \\
I_{m_{j}} & \text { otherwise }
\end{array}\right. \\
Z^{(j)}=\omega_{j}\left(Y^{(j)}\right)^{-1}
\end{gathered}
$$

The above iteration is repeated until $\|r(x, y, z)\|<T O L$, where $r(x, y, z)$ is the residual and TOL is a given tolerance. One can use backtracking line search [10] or other techniques to get the stepsize $h$.

As a result of equation (4), the Infeasible Newton's method given is said to use the $\mathrm{ZY}$ search direction. In the next section, three other search directions for the method are described.

\section{Search Directions for Infeasible Newton's Method}

In this section, three other search directions for Infeasible Newton's method, namely ZY+YZ, NT and $Z^{-1}$ directions are presented. The $\mathrm{ZY}$ direction has already been given in the previous section. All these methods were introduced and used in semidefinite programming $[2,12]$.

In the ZY method, the matrix $Z^{(j)}$ in the solution of the system (2)-(6) is not symmetric. In the ZY+YZ method, equation (4) is replaced with $Z^{(j)} Y^{(j)}+Y^{(j)} Z^{(j)}=2 \omega_{j} I_{m_{j}}$. Then $R_{c}^{(j)}, r_{c}^{(j)}, E$ and $F$ changes to:

$$
R_{c}^{(j)}=\omega_{j} I_{m_{j}}-1 / 2\left(Z^{(j)} Y^{(j)}+Y^{(j)} Z^{(j)}\right)
$$

$r_{c}^{(j)}=\operatorname{vec} R_{c}^{(j)}=\operatorname{vec}\left(\omega_{j} I_{m_{j}}\right)-1 / 2\left(I_{m_{j}} \otimes Z^{(j)}+Z^{(j)} \otimes I_{m_{j}}\right) y$

$r_{c}^{(j)}=\operatorname{vec} R_{c}^{(j)}=\operatorname{vec}\left(\omega_{j} I_{m_{j}}\right)-1 / 2\left(Y^{(j)} \otimes I_{m_{j}}+I_{m_{j}} \otimes Y^{(j)}\right) z$

$E=\operatorname{diag}\left(1 / 2\left(Y^{(1)} \otimes I_{m_{1}}+I_{m_{1}} \otimes Y^{(1)}\right), \ldots, 1 / 2\left(Y^{(q)} \otimes I_{m_{q}}+I_{m_{q}} \otimes Y^{(q)}\right)\right)$

$F=\operatorname{diag}\left(1 / 2\left(I_{m_{1}} \otimes Z^{(1)}+Z^{(1)} \otimes I_{m_{1}}\right), \ldots, 1 / 2\left(I_{m_{q}} \otimes Z^{(q)}+Z^{(q)} \otimes I_{m_{q}}\right)\right)$

In the $Z^{-1}$ method, equation (4) is replaced with $Y^{(j)}=\omega_{j}\left(Z^{(j)}\right)^{-1}$. Then $R_{c}^{(j)}, r_{c}^{(j)}, E$ and $F$ become:

$$
\begin{gathered}
R_{c}^{(j)}=\omega_{j}\left(Z^{(j)}\right)^{-1}-Y^{(j)} \\
r_{c}^{(j)}=\operatorname{vec} R_{c}^{(j)}=\omega_{j} \operatorname{vec}\left(\left(Z^{(j)}\right)^{-1}\right)-\operatorname{vec}\left(Y^{(j)}\right) \\
=\omega_{j} \operatorname{vec}\left(\left(Z^{(j)}\right)^{-1}\right)-\left(I_{m_{j}} \otimes I_{m_{j}}\right) \operatorname{vec}\left(Y^{(j)}\right) \\
E=\operatorname{diag}\left(\omega_{1}\left(\left(Z^{(1)}\right)^{-1} \otimes\left(Z^{(1)}\right)^{-1}\right), \ldots, \omega_{q}\left(\left(Z^{(q)}\right)^{-1} \otimes\left(Z^{(q)}\right)^{-1}\right)\right) \\
F=\operatorname{diag}\left(I_{m_{1}} \otimes I_{m_{1}}, \ldots, I_{m_{q}} \otimes I_{m_{q}}\right)
\end{gathered}
$$

The NT method replaces $E$ and $F$ in the $Z^{-1}$ method with

$$
\begin{gathered}
E=\operatorname{diag}\left(\omega_{1}\left(\left(W^{(1)}\right)^{-1} \otimes\left(W^{(1)}\right)^{-1}\right), \ldots, \omega_{q}\left(\left(W^{(q)}\right)^{-1} \otimes\left(W^{(q)}\right)^{-1}\right)\right) \\
F=\operatorname{diag}\left(I_{m_{1}} \otimes I_{m_{1}}, \ldots, I_{m_{q}} \otimes I_{m_{q}}\right),
\end{gathered}
$$

where for each $j, W^{(j)}$ is the unique scaling matrix defined by 


$$
W^{(j)}=\left(Z^{(j)}\right)^{\frac{1}{2}}\left(\left(Z^{(j)}\right)^{\frac{1}{2}} Y^{(j)}\left(Z^{(j)}\right)^{\frac{1}{2}}\right)^{-\frac{1}{2}}\left(Z^{(j)}\right)^{\frac{1}{2}}
$$

In the case of $Z Y+Y Z, Z^{-1}$ and NT methods, both $Z^{(j)}$ and $Y^{(j)}$ are symmetric [2]. See Theorem 3.1.

Theorem 3.1 In the $Z Y+Y Z, Z^{-1}$ and NT methods, both $Z^{(j)}$ and $Y^{(j)}$ are symmetric.

Proof: Since each $A_{i}^{(j)}$ is symmetric, then by equation (2), it is clear that each $Y^{(j)}$ is symmetric. In the $Z Y+Y Z$ method, equation (4), that is, $Z^{(j)} Y^{(j)}+Y^{(j)} Z^{(j)}=2 \omega_{j} I_{m j}$ is a symmetric matrix equation. So, equations (2)-(6) and $G(x, y, z)=0$ result in a mapping $G$ for Newton's method with the same domain and range. In the $Z^{-1}$ method, equation (4), that is, $Y^{(j)}=$ $\omega_{j}\left(Z^{(j)}\right)^{-1}$ is a symmetric matrix equation. So, equations (2)(6) and $G(x, y, z)=0$ result in a mapping $G$ for Newton's method having the same domain and range. Hence, application of Newton's method in $\mathrm{ZY}+\mathrm{YZ}$ and $Z^{-1}$ methods leads to symmetric interates $Z^{(j)}$. The proof of symmetry of $Z^{(j)}$ in the NT method follows from Theorem 3.1 in a paper given by Todd et al. [12].

\section{Numerical Experiments}

In this section, numerical experiments are done to compare $\mathrm{ZY}, \mathrm{ZY}+\mathrm{YZ}, Z^{-1}$ and NT search methods in the Infeasible Newton's method for computing weighted analytic center.

Table 1 describes the 35 random test problems used for our numerical experiments. These are the same test problems used by Jibrin in a 2015 paper [4], with the exception of Problem 35. The second column of Table 1 gives the dimension $n$ of the ambient space and the third column is the number $q$ of LMI constraints. The dimensions $m_{j}$ of the matrices are given in the fourth column. For each problem, $n$, $q$ and $m_{j}$ are random integers in the intervals $[2,30],[1,10]$ and $[1,5]$ respectively. For each $j$, the LMI $A_{0}^{(j)}+\sum_{i=1}^{n} x_{i} A_{i}^{(j)} \succeq 0$ was generated randomly as follows: $A_{0}^{(j)}$ is an $m_{j} \times m_{j}$ diagonal matrix with each diagonal entry chosen from $U(0,1)$. Each $A_{i}^{(j)}(1 \leq i \leq n)$ is a random $m_{j} \times m_{j}$ symmetric and sparse matrix with approximately $0.8 * m_{j}^{2}$ nonzero entries generated using the Matlab command $\operatorname{sprandsym}\left(m_{j}, 0.8\right)$. Each problem has a nonempty interior.
All codes used in our experiments were written in Matlab and ran on Dell OPTIPLEX 880 computer. Infeasible Newton's method codes for weighted analytic center was ran using the four search directions: $\mathrm{ZY}, \mathrm{ZY}+\mathrm{YZ}, Z^{-1}$ and NT. All four methods were implemented using

Table 1. Test Problems.

\begin{tabular}{|c|c|c|c|}
\hline Test Problem & $n$ & $q$ & $m$ \\
\hline 1 & 2 & 2 & {$[2,1]$} \\
\hline 2 & 3 & 4 & {$[3,4,1,2]$} \\
\hline 3 & 2 & 2 & {$[2,2]$} \\
\hline 4 & 5 & 3 & {$[4,1,3]$} \\
\hline 5 & 4 & 3 & {$[5,4,3]$} \\
\hline 6 & 4 & 5 & {$[4,3,1,1,4]$} \\
\hline 7 & 3 & 3 & {$[4,2,3]$} \\
\hline 8 & 3 & 4 & {$[4,2,2,5]$} \\
\hline 9 & 5 & 3 & {$[4,1,1]$} \\
\hline 10 & 3 & 5 & {$[5,3,5,1,4]$} \\
\hline 11 & 2 & 7 & {$[2,5,3,5,2,5,1]$} \\
\hline 12 & 5 & 6 & $[5,1,3,4,1,4]]$ \\
\hline 13 & 14 & 5 & {$[5,1,3,4,2]$} \\
\hline 14 & 20 & 5 & {$[5,2,5,1,5]$} \\
\hline 15 & 3 & 8 & {$[5,4,1,5,3,5,1,3]$} \\
\hline 16 & 9 & 7 & {$[1,4,2,4,4,2,2]$} \\
\hline 17 & 6 & 5 & {$[4,4,2,1,4]$} \\
\hline 18 & 10 & 2 & {$[3,5]$} \\
\hline 19 & 15 & 9 & {$[2,5,3,1,2,3,3,1,2]$} \\
\hline 20 & 8 & 2 & {$[4,5]$} \\
\hline 21 & 19 & 7 & {$[5,2,2,2,5,5,5]$} \\
\hline 22 & 9 & 10 & {$[3,4,1,1,3,5,5,4,5,2]$} \\
\hline 23 & 3 & 4 & {$[2,3,2,5]$} \\
\hline 24 & 8 & 2 & {$[5,1]$} \\
\hline 25 & 2 & 8 & {$[5,2,1,1,1,5,3,3]$} \\
\hline 26 & 13 & 8 & {$[4,1,4,2,3,1,2,1]$} \\
\hline 27 & 24 & 10 & {$[5,4,5,1,4,2,3,5,5,2]$} \\
\hline 28 & 5 & 6 & {$[4,1,4,2,1,3]$} \\
\hline 29 & 16 & 3 & {$[2,2,3]$} \\
\hline 30 & 2 & 2 & {$[4,5]$} \\
\hline 31 & 2 & 4 & {$[1,5,5,5]$} \\
\hline 32 & 4 & 4 & {$[5,1,4,5]$} \\
\hline 33 & 4 & 4 & {$[1,2,3,5]$} \\
\hline 34 & 17 & 9 & {$[1,5,2,1,2,5,1,4,3]$} \\
\hline 35 & 4 & 6 & {$[2,1,5,3,5,2]$} \\
\hline
\end{tabular}

Table 2. Iterations and time taken by each method to find a point in the interior of the feasible region using the given weights. The entry "*” means that Infeasible Newton's method has failed to find an interior point after the maximum number of 500 iterations or due to numerical problems.

\begin{tabular}{|c|c|c|c|c|c|c|c|c|c|}
\hline \multirow{2}{*}{ Prob } & \multirow{2}{*}{ Weights } & \multicolumn{2}{|l|}{$\mathbf{Z Y}$} & \multicolumn{2}{|l|}{$Z^{-1}$} & \multicolumn{2}{|c|}{$\mathbf{Z Y}+\mathbf{Y Z}$} & \multicolumn{2}{|l|}{ NT } \\
\hline & & Iter & Time & Iter & Time & Iter & Time & Iter & Time \\
\hline & $\Omega$ & & $(\mathrm{sec})$ & & $(\mathrm{sec})$ & & $(\mathrm{sec})$ & & $(\mathrm{sec})$ \\
\hline 1 & {$\left[10^{12}, 10\right]$} & 2 & 0.0490 & $*$ & * & 2 & 0.2311 & $*$ & $*$ \\
\hline 2 & {$\left[10^{12}, 100,100,1\right]$} & 3 & 0.0271 & $*$ & $*$ & 3 & 0.0280 & * & $*$ \\
\hline 3 & {$\left[10^{12}, 1000\right]$} & 51 & 0.2100 & * & $*$ & 48 & 0.2970 & * & $*$ \\
\hline 4 & {$\left[10^{12}, 10,1\right]$} & 20 & 0.1644 & $*$ & $*$ & 17 & 0.1550 & 18 & 0.4929 \\
\hline 5 & {$\left[10^{12}, 1,10\right]$} & 2 & 0.0167 & $*$ & $*$ & 2 & 0.0130 & $*$ & $*$ \\
\hline 6 & {$\left[1,10^{12}, 1,10,100\right]$} & $*$ & * & $*$ & $*$ & $*$ & * & 2 & 0.1377 \\
\hline 7 & {$\left[100,10,10^{12}\right]$} & 129 & 2.2922 & $*$ & $*$ & $*$ & $*$ & $*$ & $*$ \\
\hline
\end{tabular}




\begin{tabular}{|c|c|c|c|c|c|c|c|c|c|}
\hline \multirow{2}{*}{ Prob } & \multirow{2}{*}{ Weights } & \multicolumn{2}{|l|}{$\overline{Z Y}$} & \multicolumn{2}{|l|}{$Z^{-1}$} & \multicolumn{2}{|c|}{$\mathbf{Z Y}+\mathbf{Y Z}$} & \multicolumn{2}{|l|}{ NT } \\
\hline & & Iter & Time & Iter & Time & Iter & Time & Iter & Time \\
\hline 8 & {$\left[1,1000,10^{12}, 10\right]$} & $*$ & * & $*$ & $*$ & $*$ & $*$ & $*$ & $*$ \\
\hline 9 & {$\left[10^{12}, 1000,1000\right]$} & 1 & 0.0089 & 1 & 0.0040 & 1 & 0.0071 & * & $*$ \\
\hline 10 & {$\left[10^{12}, 1000,100,1000,100\right]$} & 6 & 0.1059 & $*$ & $*$ & 6 & 0.0612 & 52 & 2.8783 \\
\hline 11 & {$\left[10^{12}, 10,100,1000,1000,10,100\right]$} & * & $*$ & $*$ & * & * & * & $*$ & $*$ \\
\hline 12 & {$\left[10^{12}, 1,100,10,1,10\right]$} & 3 & 0.0480 & $*$ & $*$ & 3 & 0.0338 & $*$ & $*$ \\
\hline 13 & {$\left[1000,10^{12}, 1000,100,100\right]$} & $*$ & $*$ & $*$ & $*$ & $*$ & $*$ & $*$ & $*$ \\
\hline 14 & {$\left[1,1000,10^{12}, 1000,1\right]$} & * & $*$ & $*$ & * & $*$ & * & $*$ & $*$ \\
\hline 15 & {$\left[1,1,1,100,10^{12}, 100,100,10\right]$} & $*$ & * & $*$ & $*$ & $*$ & $*$ & $*$ & $*$ \\
\hline 16 & {$[10,1,10,1000,1000,10,1]$} & 24 & 0.3132 & $*$ & * & 23 & 0.2861 & $*$ & $*$ \\
\hline 17 & {$[100,100,10,1,1000]$} & 3 & 0.0316 & $*$ & $*$ & 3 & 0.0275 & $*$ & $*$ \\
\hline 18 & {$[1,1000]$} & 2 & 0.2249 & $*$ & $*$ & 2 & 0.0098 & 130 & 0.5293 \\
\hline 19 & {$[100,10,1000,1,1000,100,100,1000,10]$} & 10 & 0.1678 & * & * & 10 & 0.1590 & * & * \\
\hline 20 & {$[1000,1000]$} & 1 & 0.0084 & 1 & 0.0086 & 1 & 0.0061 & $*$ & $*$ \\
\hline 21 & {$[1,1,10,1,100,10,1]$} & 21 & 0.3363 & $*$ & $*$ & 21 & 0.3405 & $*$ & * \\
\hline 22 & {$[1,1000,100,10,1000,1,100,100,100,1]$} & 7 & 0.1721 & $*$ & * & 4 & 0.0917 & $*$ & * \\
\hline 23 & {$[100,10,1,1]$} & 4 & 0.0344 & $*$ & $*$ & 4 & 0.0292 & 5 & 0.0614 \\
\hline 24 & {$[1000,1]$} & 12 & 0.0536 & * & * & 11 & 0.0423 & * & * \\
\hline 25 & {$[1,1,10,1,1000,1,1,10]$} & 8 & 0.1268 & $*$ & $*$ & 9 & 0.1312 & $*$ & $*$ \\
\hline 26 & {$[100,1,100,1,1,1,1,1]$} & 20 & 0.2687 & $*$ & $*$ & 20 & 0.2713 & $*$ & $*$ \\
\hline 27 & {$[1,1,1,10,1,10,1,100,1,10]$} & 8 & 0.2345 & $*$ & $*$ & 10 & 0.2790 & $*$ & * \\
\hline 28 & {$[10,10,100,1,1,1]$} & 7 & 0.0773 & * & $*$ & 7 & 0.0672 & * & $*$ \\
\hline 29 & {$[1,1,10]$} & 1 & 0.0122 & 1 & 0.0043 & 1 & 0.0077 & 1 & 0.0061 \\
\hline 30 & {$[10,1]$} & 2 & 0.0147 & $*$ & $*$ & 2 & 0.0098 & $*$ & $*$ \\
\hline 31 & {$[1,10,1,1]$} & 1 & 0.0136 & 1 & 0.0120 & 1 & 0.0098 & $*$ & $*$ \\
\hline 32 & {$[100,1,10,1]$} & 8 & 0.0759 & * & $*$ & 8 & 0.0647 & 11 & 0.1767 \\
\hline 33 & {$[100,100,1,10]$} & 6 & 0.0495 & $*$ & $*$ & 6 & 0.0414 & 2 & 0.0116 \\
\hline 34 & {$[1,100,1,1,1,10,100,100,10]$} & 7 & 0.1372 & $*$ & $*$ & 8 & 0.1404 & $*$ & $*$ \\
\hline 35 & {$[1,1,100,1,10,1]$} & 2 & 0.0277 & $*$ & $*$ & 2 & 0.0246 & $*$ & $*$ \\
\hline
\end{tabular}

Table 3. Iterations and time taken by each method to find the weighted analytic center using the given weights. The entry “ $\star$ ” means that Infeasible Newton's method has failed to find the weighted analytic center due to numerical problems or after the maximum number of 500 iterations.

\begin{tabular}{|c|c|c|c|c|c|c|c|c|c|}
\hline \multirow{2}{*}{ Prob } & \multirow{2}{*}{ Weights } & \multicolumn{2}{|l|}{ ZY } & \multicolumn{2}{|l|}{$Z^{-1}$} & \multicolumn{2}{|c|}{$\mathbf{Z Y}+\mathbf{Y Z}$} & \multicolumn{2}{|l|}{ NT } \\
\hline & & Iter & Time & Iter & Time & Iter & Time & Iter & Time \\
\hline & $\Omega$ & & $(\mathrm{sec})$ & & $(\mathrm{sec})$ & & $(\mathrm{sec})$ & & $(\mathrm{sec})$ \\
\hline 1 & {$\left[10^{12}, 10\right]$} & 6 & 0.0590 & $*$ & $*$ & 8 & 0.2883 & * & * \\
\hline 2 & {$\left[10^{12}, 100,100,1\right]$} & * & $*$ & $*$ & $*$ & * & $*$ & $*$ & $*$ \\
\hline 3 & {$\left[\left[10^{12}, 1000\right]\right.$} & 97 & 0.3827 & $*$ & $*$ & 93 & 0.5589 & $*$ & $*$ \\
\hline 4 & {$\left[10^{12}, 10,1\right]$} & $*$ & $*$ & $*$ & $*$ & $*$ & * & * & * \\
\hline 5 & {$\left[10^{12}, 1,10\right]$} & * & $*$ & $*$ & $*$ & * & $*$ & $*$ & $*$ \\
\hline 6 & {$\left[1,10^{12}, 1,10,100\right]$} & * & $*$ & $*$ & $*$ & $*$ & * & $*$ & $*$ \\
\hline 7 & {$\left[100,10,10^{12}\right]$} & * & $*$ & $*$ & $*$ & * & * & $*$ & * \\
\hline 8 & {$\left[1,1000,10^{12}, 10\right]$} & * & $*$ & $*$ & $*$ & * & $*$ & $*$ & $*$ \\
\hline 9 & {$\left[10^{12}, 1000,1000\right]$} & * & $*$ & * & $*$ & * & * & $*$ & * \\
\hline 10 & {$\left[10^{12}, 1000,100,1000,100\right]$} & * & $*$ & $*$ & * & * & * & $*$ & * \\
\hline 11 & {$\left[10^{12}, 10,100,1000,1000,10,100\right]$} & * & $*$ & * & $*$ & * & * & $*$ & * \\
\hline 12 & {$\left[10^{12}, 1,100,10,1,10\right]$} & * & $*$ & $*$ & $*$ & * & * & $*$ & * \\
\hline 13 & {$\left[1000,10^{12}, 1000,100,100\right]$} & * & $*$ & $*$ & $*$ & * & * & $*$ & $*$ \\
\hline 14 & {$\left[1,1000,10^{12}, 1000,1\right]$} & * & $*$ & $*$ & $*$ & $*$ & * & * & * \\
\hline 15 & {$\left[1,1,1,100,10^{12}, 100,100,10\right]$} & * & $*$ & $*$ & * & $*$ & * & $*$ & * \\
\hline 16 & {$[10,1,10,1000,1000,10,1]$} & 27 & 0.3412 & $*$ & * & 26 & 0.3146 & $*$ & * \\
\hline 17 & {$[100,100,10,1,1000]$} & 7 & 0.0612 & * & * & 7 & 0.0568 & $*$ & * \\
\hline 18 & $[1,1000]]$ & 8 & 0.2463 & * & * & 7 & 0.0273 & $*$ & * \\
\hline 19 & {$[100,10,1000,1,1000,100,100,1000,10]$} & 17 & 0.2600 & $*$ & * & 18 & 0.2690 & $*$ & * \\
\hline 20 & {$[1000,1000]$} & 5 & 0.0254 & $*$ & * & 5 & 0.0206 & $*$ & * \\
\hline 21 & {$[1,1,10,1,100,10,1]$} & 24 & 0.3748 & $*$ & * & 23 & 0.3687 & $*$ & * \\
\hline 22 & {$[1,1000,100,10,1000,1,100,100,100,1]$} & 10 & 0.2307 & $*$ & * & 10 & 0.2137 & $*$ & * \\
\hline 23 & {$[100,10,1,1]$} & 9 & 0.0619 & $*$ & * & 8 & 0.0511 & $*$ & * \\
\hline 24 & {$[1000,1]$} & 14 & 0.0602 & $*$ & * & 13 & 0.0487 & $*$ & * \\
\hline 25 & {$[1,1,10,1,1000,1,1,10]$} & 11 & 0.1606 & $*$ & $*$ & 11 & 0.1542 & $*$ & $*$ \\
\hline 26 & {$[100,1,100,1,1,1,1,1]$} & 22 & 0.2893 & $*$ & $*$ & 22 & 0.2922 & $*$ & $*$ \\
\hline 27 & {$[1,1,1,10,1,10,1,100,1,10]$} & 12 & 0.3293 & $*$ & * & 13 & 0.3511 & $*$ & * \\
\hline 28 & {$[10,10,100,1,1,1]$} & 10 & 0.1016 & $*$ & * & 9 & 0.0836 & $*$ & * \\
\hline 29 & {$[1,1,10]$} & $*$ & $*$ & $*$ & $*$ & * & $*$ & $*$ & $*$ \\
\hline 30 & {$[10,1]$} & 6 & 0.0304 & $*$ & $*$ & 6 & 0.0246 & $*$ & * \\
\hline 31 & {$[1,10,1,1]$} & 5 & 0.0458 & 149 & 1.5269 & 4 & 0.0335 & $*$ & $*$ \\
\hline
\end{tabular}




\begin{tabular}{|c|c|c|c|c|c|c|c|c|c|}
\hline \multirow{2}{*}{ Prob } & \multirow{2}{*}{ Weights } & \multicolumn{2}{|l|}{$\mathbf{Z Y}$} & \multicolumn{2}{|l|}{$Z^{-1}$} & \multicolumn{2}{|c|}{$Z \mathbf{Z Y}+\mathrm{YZ}$} & \multicolumn{2}{|l|}{ NT } \\
\hline & & Iter & Time & Iter & Time & Iter & Time & Iter & Time \\
\hline 32 & {$[100,1,10,1]$} & 12 & 0.1051 & $*$ & * & 11 & 0.0851 & $*$ & * \\
\hline 33 & {$[100,100,1,10]$} & 9 & 0.0668 & $*$ & $*$ & 10 & 0.0639 & $*$ & $*$ \\
\hline 34 & {$[1,100,1,1,1,10,100,100,10]$} & 10 & 0.1726 & $*$ & $*$ & 11 & 0.1817 & $*$ & $*$ \\
\hline 35 & {$[1,1,100,1,10,1]$} & 6 & 0.0390 & $*$ & $*$ & 6 & 0.0607 & $*$ & $*$ \\
\hline
\end{tabular}

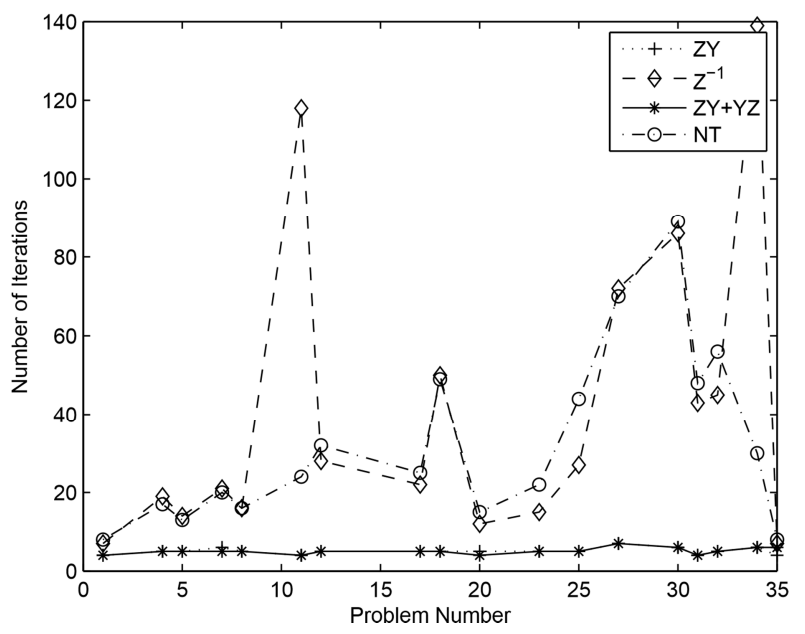

Figure 1. Problem Number VS Iterations needed to find analytic center for the 18 problems where all four methods were successful. $+=Z Y, \diamond=Z^{-1}$, $*=Z Y+Y Z, o=N T$.

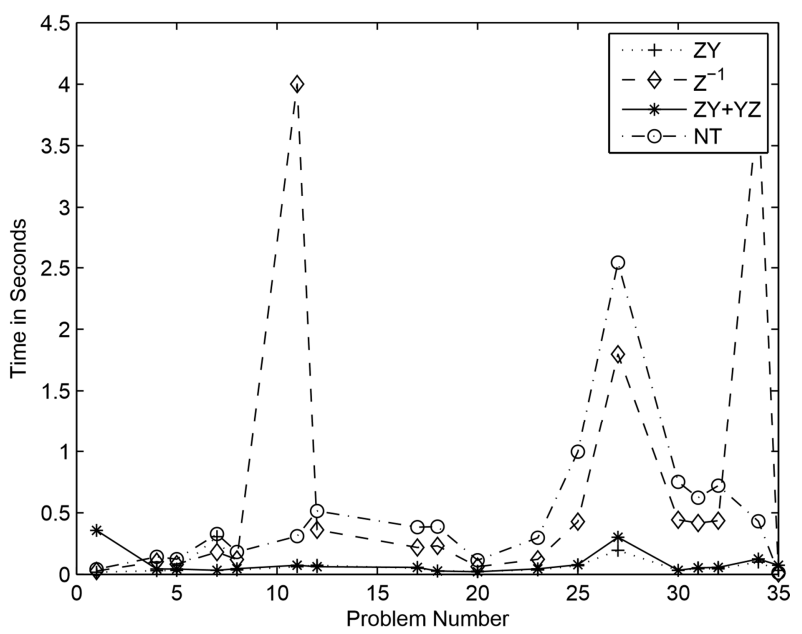

Figure 2. Problem Number VS Time needed to find analytic center for the 18 problems where all four methods were successful. $+=Z Y, \diamond=Z^{-1}$, $*=Z Y+Y Z, o=N T$.
Table 4. Number of problems out of 35, where the given method was successful from the results in Table 2 and Table 3.

\begin{tabular}{lll}
\hline \multirow{2}{*}{ Method } & Finding Interior Point & Finding Weighted Analytic Center \\
\cline { 2 - 3 } & Success Number & Success Number \\
\hline ZY & 29 & 21 \\
$Z^{-1}$ & 4 & 1 \\
$Z$ ZY+YZ & 28 & 21 \\
NT & 8 & 0 \\
\hline
\end{tabular}

a tolerance of $T O L=10^{-4}$ and up to a maximum of 500 iterations. The starting point is infeasible and random such that each of its components is chosen from a normal distribution with mean 0 and variance $10^{6}$. Backtracking line search technique is used in each of the four methods. Table 2 and Table 3 give the results of our experiments, where one of the weights is relatively very large in Problems 1-15. In Problems 16-35, one of the weights is relatively large, but not very large. Table 2 gives the number of iterations and time to find an interior point of the feasible region from the starting point. In Table 2, the entry " $\star$ " means that Infeasible Newton's method has failed to find an interior point after the maximum number of 500 iterations or due to numerical problems. We see that $\mathrm{ZY}+\mathrm{YZ}$ takes the least number of iterations on average and $Z Y$ takes the least time on average. $\mathrm{ZY}+\mathrm{YZ}$ and $\mathrm{ZY}$ are the best methods, followed by NT and then $Z^{-1}$. Table 3 gives the number of iterations and time to find the weighted analytic center. In Table 3 , the entry " $\star$ " means that Infeasible Newton's method has failed to find the weighted analytic center due to numerical problems or after the maximum number of 500 iterations. Table 4 summarizes the results of Table 2 and Table 3. The results in Table 4 show that $\mathrm{ZY}+\mathrm{YZ}$ and $\mathrm{ZY}$ were the most successful while both $Z^{-1}$ and NT failed to work well. In Table 5 and Table 6, the results are given where each of the weights is 1 . In this case, the weighted analytic center is simply called the analytic center.

Table 5. Iterations and time taken by each method to find a point in the interior of the feasible region using the given the weight $\omega=[1,1, \ldots, 1]$. The entry “ * " means that Infeasible Newton's method has failed to find an interior point after the maximum number of 500 iterations or due to numerical problems.

\begin{tabular}{|c|c|c|c|c|c|c|c|c|}
\hline \multirow{2}{*}{ Problem } & \multicolumn{2}{|l|}{$\mathbf{Z Y}$} & \multicolumn{2}{|l|}{$Z^{1}$} & \multicolumn{2}{|c|}{$\mathrm{ZY}+\mathrm{YZ}$} & \multicolumn{2}{|l|}{ NT } \\
\hline & Iter & Time & Iter & Time & Iter & Time & Iter & Time \\
\hline & & $(\mathrm{sec})$ & & (sec) & & $(\mathrm{sec})$ & & (sec) \\
\hline 1 & 1 & 0.0089 & 1 & 0.0073 & 1 & 0.3195 & 1 & 0.0079 \\
\hline 2 & 2 & 0.0177 & 2 & 0.0188 & 2 & 0.0213 & 2 & 0.0168 \\
\hline 3 & 1 & 0.0070 & 1 & 0.0055 & 1 & 0.0088 & 1 & 0.0078 \\
\hline 4 & 2 & 0.0166 & 2 & 0.0151 & 2 & 0.0218 & 2 & 0.0191 \\
\hline 5 & 1 & 0.0103 & 1 & 0.0097 & 1 & 0.0119 & 1 & 0.0116 \\
\hline 6 & 1 & 0.0133 & 1 & 0.0134 & 1 & 0.0156 & 1 & 0.0090 \\
\hline 7 & 1 & 0.2494 & 1 & 0.0762 & 1 & 0.0091 & 1 & 0.1367 \\
\hline 8 & 1 & 0.0117 & 1 & 0.0136 & 1 & 0.0126 & 1 & 0.0253 \\
\hline 9 & 1 & 0.2908 & 1 & 0.0773 & 1 & 0.0095 & 1 & 0.0489 \\
\hline 10 & 1 & 0.0070 & 1 & 0.0068 & 1 & 0.0164 & 1 & 0.0159 \\
\hline 11 & 1 & 0.0201 & 1 & 0.0169 & 1 & 0.0219 & 1 & 0.0115 \\
\hline
\end{tabular}




\begin{tabular}{|c|c|c|c|c|c|c|c|c|}
\hline \multirow{2}{*}{ Problem } & \multicolumn{2}{|l|}{$\mathrm{ZY}$} & \multicolumn{2}{|l|}{$\boldsymbol{Z}^{I}$} & \multicolumn{2}{|c|}{$\mathbf{Z Y}+\mathbf{Y Z}$} & \multicolumn{2}{|l|}{ NT } \\
\hline & Iter & Time & Iter & Time & Iter & Time & Iter & Time \\
\hline 12 & 2 & 0.0458 & 2 & 0.0613 & 2 & 0.0295 & 2 & 0.0469 \\
\hline 13 & 2 & 0.0293 & 3 & 0.0435 & 2 & 0.0359 & 2 & 0.0146 \\
\hline 14 & 13 & 0.1498 & $*$ & $*$ & 13 & 0.1876 & 24 & 0.1567 \\
\hline 15 & 1 & 0.0237 & 1 & 0.0235 & 1 & 0.0239 & 1 & 0.0222 \\
\hline 16 & 2 & 0.0446 & 2 & 0.0375 & 2 & 0.0423 & 2 & 0.0181 \\
\hline 17 & 2 & 0.0256 & 2 & 0.0246 & 2 & 0.0273 & 2 & 0.0325 \\
\hline 18 & 2 & 0.0151 & 2 & 0.0144 & 2 & 0.0132 & 2 & 0.0184 \\
\hline 19 & 3 & 0.0559 & 2 & 0.0448 & 3 & 0.0579 & $*$ & * \\
\hline 20 & 1 & 0.0087 & 1 & 0.0084 & 1 & 0.0084 & 1 & 0.0092 \\
\hline 21 & 12 & 0.2475 & 10 & 0.1670 & 12 & 0.2669 & $*$ & $*$ \\
\hline 22 & 2 & 0.0553 & $*$ & $*$ & 2 & 0.0605 & 2 & 0.0703 \\
\hline 23 & 1 & 0.0111 & 1 & 0.0121 & 1 & 0.0124 & 1 & 0.0149 \\
\hline 24 & 4 & 0.0229 & $*$ & $*$ & 5 & 0.0349 & 36 & 0.3229 \\
\hline 25 & 2 & 0.0346 & 2 & 0.0394 & 2 & 0.0363 & 2 & 0.0462 \\
\hline 26 & 13 & 0.4362 & $*$ & $*$ & 13 & 0.2624 & 22 & 0.5945 \\
\hline 27 & 3 & 0.1036 & 2 & 0.0788 & 3 & 0.1366 & 3 & 0.1310 \\
\hline 28 & 3 & 0.0375 & 3 & 0.0388 & 3 & 0.0419 & 3 & 0.0450 \\
\hline 29 & 1 & 0.0116 & 1 & 0.0039 & 1 & 0.0115 & 1 & 0.0053 \\
\hline 30 & 2 & 0.0145 & 2 & 0.0140 & 2 & 0.0131 & 2 & 0.0201 \\
\hline 31 & 1 & 0.0223 & 1 & 0.0130 & 1 & 0.0159 & 1 & 0.0157 \\
\hline 32 & 2 & 0.0222 & 2 & 0.0233 & 2 & 0.0257 & 2 & 0.0286 \\
\hline 33 & 4 & 0.0349 & $*$ & $*$ & 4 & 0.0461 & 24 & 0.2680 \\
\hline 34 & 2 & 0.0453 & 2 & 0.0464 & 2 & 0.0524 & 2 & 0.0276 \\
\hline 35 & 1 & 0.0270 & 1 & 0.0026 & 2 & 0.0300 & 1 & 0.0018 \\
\hline
\end{tabular}

Table 6. Iterations and time taken by each method to find the analytic center that is, using the given the weight $\omega=[1,1, \ldots, 1]$. The entry “ $\star$ " means that Infeasible Newton's method has failed to find the analytic center due to numerical problems or after the maximum number of 500 iterations.

\begin{tabular}{|c|c|c|c|c|c|c|c|c|}
\hline \multirow{2}{*}{ Problem } & \multicolumn{2}{|l|}{$\mathbf{Z Y}$} & \multicolumn{2}{|l|}{$\boldsymbol{Z}^{I}$} & \multicolumn{2}{|c|}{$\overline{Z Y+Y Z}$} & \multicolumn{2}{|l|}{ NT } \\
\hline & Iter & Time & Iter & Time & Iter & Time & Iter & Time \\
\hline & & $(\mathrm{sec})$ & & $(\mathrm{sec})$ & & $(\mathrm{sec})$ & & $(\mathrm{sec})$ \\
\hline 1 & 4 & 0.0181 & 7 & 0.0296 & 4 & 0.3564 & 8 & 0.0433 \\
\hline 2 & 6 & 0.0404 & $*$ & $*$ & 6 & 0.0534 & 47 & 0.4401 \\
\hline 3 & 10 & 0.0381 & $*$ & * & 9 & 0.0509 & 19 & 0.3052 \\
\hline 4 & 5 & 0.0299 & 19 & 0.1036 & 5 & 0.0424 & 17 & 0.1428 \\
\hline 5 & 5 & 0.0307 & 14 & 0.0831 & 5 & 0.0428 & 13 & 0.1241 \\
\hline 6 & 5 & 0.0435 & $*$ & $*$ & 5 & 0.0575 & 29 & 0.2544 \\
\hline 7 & 6 & 0.3064 & 21 & 0.1810 & 5 & 0.0331 & 20 & 0.3278 \\
\hline 8 & 5 & 0.0352 & 16 & 0.1205 & 5 & 0.0465 & 16 & 0.1821 \\
\hline 9 & 5 & 0.3294 & $*$ & $*$ & 5 & 0.0312 & 62 & 0.4482 \\
\hline 10 & 7 & 0.0399 & 85 & 0.5903 & 5 & 0.0635 & 70 & 0.5882 \\
\hline 11 & 4 & 0.0618 & 118 & 4.0018 & 4 & 0.0734 & 24 & 0.3111 \\
\hline 12 & 5 & 0.0743 & 28 & 0.3619 & 5 & 0.0626 & 32 & 0.5154 \\
\hline 13 & 6 & 0.0638 & $*$ & $*$ & 6 & 0.0850 & 50 & 0.4806 \\
\hline 14 & 17 & 0.1873 & * & * & 16 & 0.2343 & 101 & 0.7694 \\
\hline 15 & 6 & 0.1046 & $*$ & * & 5 & 0.0999 & 87 & 1.8125 \\
\hline 16 & 6 & 0.0886 & $*$ & * & 6 & 0.0919 & 32 & 0.4146 \\
\hline 17 & 5 & 0.0484 & 22 & 0.2176 & 5 & 0.0564 & 25 & 0.3839 \\
\hline 18 & 5 & 0.0277 & 50 & 0.2313 & 5 & 0.0266 & 49 & 0.3879 \\
\hline 19 & 7 & 0.1060 & $*$ & $*$ & 7 & 0.1317 & $*$ & $*$ \\
\hline 20 & 5 & 0.0258 & 12 & 0.0627 & 4 & 0.0219 & 15 & 0.1141 \\
\hline 21 & 16 & 0.3230 & $*$ & $*$ & 16 & 0.3482 & $*$ & $*$ \\
\hline 22 & 6 & 0.1344 & $*$ & * & 6 & 0.1682 & 79 & 3.5432 \\
\hline 23 & 5 & 0.0355 & 15 & 0.1218 & 5 & 0.0439 & 22 & 0.2976 \\
\hline 24 & 8 & 0.0377 & $*$ & $*$ & 8 & 0.0503 & $*$ & $*$ \\
\hline 25 & 5 & 0.0707 & 27 & 0.4286 & 5 & 0.0777 & 44 & 0.9968 \\
\hline 26 & 16 & 0.4689 & $*$ & $*$ & 16 & 0.3070 & 44 & 1.0047 \\
\hline 27 & 7 & 0.1946 & 72 & 1.7975 & 7 & 0.3018 & 70 & 2.5422 \\
\hline 28 & 6 & 0.0624 & $*$ & $*$ & 6 & 0.0733 & 55 & 0.2527 \\
\hline 29 & * & * & * & * & * & * & * & * \\
\hline 30 & 6 & 0.0325 & 86 & 0.4434 & 6 & 0.0323 & 89 & 0.7516 \\
\hline 31 & 4 & 0.0460 & 43 & 0.4163 & 4 & 0.0535 & 48 & 0.6225 \\
\hline 32 & 5 & 0.0449 & 45 & 0.4357 & 5 & 0.0559 & 56 & 0.7213 \\
\hline 33 & 8 & 0.0593 & $*$ & $*$ & 8 & 0.0837 & $*$ & $*$ \\
\hline 34 & 6 & 0.0998 & 139 & 3.8625 & 6 & 0.1242 & 30 & 0.4312 \\
\hline 35 & 4 & 0.0311 & 7 & 0.0079 & 6 & 0.0740 & 8 & 0.0128 \\
\hline
\end{tabular}


Table 7. Number of problems out of 35, where method was successful from the results in Table 5 and Table 6.

\begin{tabular}{lll}
\hline \multirow{2}{*}{ Method } & Finding Interior Point & Finding Analytic Center \\
\cline { 2 - 3 } & Success Number & Success Number \\
\hline ZY & 35 & 34 \\
$Z^{-1}$ & 30 & 19 \\
ZY+YZ & 35 & 34 \\
NT & 33 & 30 \\
\hline
\end{tabular}

Table 5 gives the number of iterations and time to find an interior point of the feasible region with each weight set as 1 . It shows that $\mathrm{ZY}+\mathrm{YZ}$ and $\mathrm{ZY}$ are the best methods in finding an interior in terms of iterations and time, followed by NT, and then by $Z^{-1}$. Table 6 gives the number of iterations and time to find the analytic center. Table 7 summarizes the results of Table 5 and Table 6 . Note all the four methods were successful in finding the analytic center in 18 of the 35 test problems. Figure 1 shows the graph of iterations vs. the 18 problems. The graph of time vs. the 18 problems is given in Figure 2. Figure 1, Figure 2 and the results in Table 7 show that $\mathrm{ZY}+\mathrm{YZ}$ and $\mathrm{ZY}$ are the best methods, followed by NT, and then $Z^{-1}$.

\section{Conclusion}

Four search direction methods for Infeasible Newton's method for computing weighted analytic center for linear matrix inequalities, namely: $\mathrm{ZY}, \mathrm{ZY}+\mathrm{YZ}, Z^{-1}$ and NT methods are presented and compared. The four search directions $Z Y, Z Y+Y Z, Z^{-1}$ and NT methods have been used in the problem of semidefinite programming.

Randomly generated test problems are used to compare the four methods. Our numerical results indicate that the $Z Y+Y Z$ and $\mathrm{ZY}$ methods converge more rapidly and they handle weights better compared to the other methods, when some of the weights are very large relative to the other weights. $\mathrm{ZY}+\mathrm{YZ}$ took the least number of iterations on average and is closely followed by ZY, then NT and then $Z^{-1}$ methods. ZY also took the least time among the other four methods.

Backtracking line search is used in our experiments. It would be of interest to study and compare the effect of other line search methods on the four methods. We hope in future to compare the performance of the four methods on weighted analytic center for second order cone constraints.

\section{Acknowledgements}

This research work was initially started during my sabbatical leave at Jubail University College in Saudi Arabia.

\section{References}

[1] F. Alizadeh, "Interior Point Methods in Semidefinite Programming with Applications to Combinatorial Optimization", SIAM Journal on Optimization, Vol. 5, No. 1, 1995, pp. 13-51.

[2] F. Alizadeh, J. A. Haeberly and M. Overton, "Primal-Dual Methods for Semidefinite Programming: Convergence Rates, Stability and Numerical Results", SIAM Journal on Optimization, Vol. 8, 1998, no. 3, pp. 746-768.

[3] D. S. Atkinson and P. M. Vaidya, "A scaling technique for finding the weighted analytic center of a polytope," Math. Prog., 57, 1992, pp. 163-192.

[4] S. Jibrin, "Computing Weighted Analytic Center for Linear Matrix Inequalities Using Infeasible Newton's Method", Journal of Mathematics, vol. 2015, Article ID 456392, 2015.

[5] S. Jibrin and J. W. Swift, "The Boundary of the Weighted Analytic Center for Linear Matrix inequalities." Journal of Inequalities in Pure and Applied Mathematics, Vol. 5, Issue 1, Article 14, 2004.

[6] J. Machacek and S. Jibrin, "An Interior Point Method for Solving Semidefinite Programs Using Cutting Planes and Weighted Analytic Centers", Journal of Applied Mathematics, Vol. 2012, Article ID 946893, 2012.

[7] I. S. Pressman and S. Jibrin, "A Weighted Analytic Center for Linear Matrix Inequalities", Journal of Inequalities in Pure and Applied Mathematics, Vol. 2, Issue 3, Article 29, 2002.

[8] J. Renegar, “A polynomial-time algorithm, based on Newton's method, for linear programming," Math. Programming, Vol. 40, 1988, pp. 59-93.

[9] L. Vandenberghe and S. Boyd, "Semidefinite Programming", SIAM Review, Vol. 38, 1996, pp. 49-95.

[10] L. Vandenberghe and S. Boyd, "Convex Optimization", Cambridge University Press, New York 2004.

[11] L. Vandenberghe, S.-P. Boyd and S. Wu, "Determinant Maximization with Linear Matrix Inequality Constraints", SIAM Journal on Matrix Analysis, Vol. 19, no. 2, 1998, pp. 499-533.

[12] M. J. Todd, K. C. Toh and R. H. Tuntuncu, "On the NesterovTodd direction in semidefinite programming," SIAM $J$. Optim., vol. 8, 1998, pp. 769-796. 